\title{
Plasma Levels of Leukotriene B4 and Prostaglandin E2 Correlation with Endoscopic Changes after NSAID Gastropathies Pantoprazole Treatment in Patients with Cardiovascular Pathology
}

\author{
Maher Mbarki ${ }^{1}$, Helen Sklyarova ${ }^{1}$, Krystyna Aksentiychuk $^{1}$, Ihor Tumak ${ }^{2}$ and Eugene Sklyarov ${ }^{1}$ \\ 1. Department of Therapy No.1 and Medical Diagnostics, Lviv National Medical University Named After Danylo Halytsky, Lviv \\ 79010, Ukraine \\ 2. Department of Endoscopy, Lviv Clinical Municipal Communal Emergency Hospital, Lviv 79059, Ukraine
}

\begin{abstract}
The article describes the pantoprazole healing effect on the gastroduodenal mucosa in patients with NSAID gastropathy. Two groups of patients were compared, depending on the treatment they have got group, which was taking $75 \mathrm{mg}$ of enteric aspirin per day and the group, where pantoprazole has been added to aspirin in the usual dose. Mucosa assessment was studied using fibrogastroduodenoscopy and Lanza score. Also, the relationship between aggression factors that cause NSAID gastropathy and mucosal protection agents has been studied. It was proved that the pantoprazole influence reduces the ulcerative-erosive lesions amount. Stomach erosive lesions percentage decreased from $58.18 \%$ to $42.42 \%$, stomach ulcers from $14.55 \%$ to $6.06 \%$, duodenum erosive lesions decreased from $34.55 \%$ to $24.24 \%$, duodenum ulcers deceased from $9.09 \%$ to $3.03 \%$. A positive correlation between LTB4 and Lanza scale was checked after pantoprazole treatment, indicating an impact on the LTB4 reduction in ulcers healing in patients with NSAID gastropathy.
\end{abstract}

Key words: Aspirin, NSAIDs, pantoprazole, LTB4, PGE2.

\section{Introduction}

NSAIDs (nonsteroidal anti-inflammatory drugs) are widely used for the prevention of cardiovascular diseases. Low-dose aspirin and other cyclooxygenase inhibitors reduce the risk of CHD (coronary heart disease) complications and are the important factors in the primary prevention of cardiovascular pathology [1].

However, depending on the dose and NSAIDs duration, there may be development of the stomach and duodenum mucosa lesions in the erosions form and ulceration even [2]. Therefore, the problem of preventing the development of such gastropathiesis becomes increasingly important. Erosive gastroduodenal lesions are found in $15 \sim 30 \%$ of

Corresponding author: Eugene Sklyarov, M.D., professor, research fields: gastroenterology and internal medicine. patients who were prescribed NSAIDs [3].

Risk of ulceration increases in patients with coronary heart disease mainly aged 65 years, who have to take ASA (acetylsalicylic acid) every day. In such cases, for the prevention purposes, proton pump inhibitors, $\mathrm{H}_{2_{-}}$histamine receptor blockers or misoprostol are prescribed [4].

Currently, NSAIDs gastropathies are diagnosed using the stomach and duodenum endoscopy, due to the fact that approximately $61 \%$ of the pathology proceeds asymptomatically [5]. Among the endoscopic criteria, a clear connection between erosions and ulcers occurrence and NSAIDs taking is important [6]. The most characteristic lesions during protracted administration of ASA are superficial erosions with the bottom covered with fibrin. Multiple lesions are mainly localized in the antrum, but heal after discontinuation 
of NSAIDs [7].

Preventing adverse effects with NSAIDs called gastrocytoprotection aimed at the stomach protection, where the PPIs (proton pump inhibitors) are used to play the main role [8].

Leukotriene B4 and prostaglandin E2 are the most important factors involved in processes of the aggression and protection the stomach and duodenum mucous membrane. In physiological conditions, there is a balance between the production of its arachidonic acid derivatives [9].

Appointment of NSAIDs in patients with cardiovascular disorders for the thrombosis and embolism prevention leads to cyclooxygenase inhibition by changing the balance between PgE2 and LTB4 in favor of the latter with NSAID gastropathy development [10].

The method of erosive and ulcerative gastroduodenal lesions prevention is the PPIs using. There was found a significant decrease of erosions and ulcers after a pantoprazole course assignment in the treatment of NSAID gastropathies [11].

The aim of this study was to investigate the impact of pantoprazole on NSAIDs gastropathies healing in patients with coronary heart disease who have been taking aspirin for a longtime period.

\section{Materials and Methods}

The study involved 70 patients with coronary heart disease, who were hospitalized in therapeutic department of Municipal City Clinical Emergency Hospital of Lvov, Ukraine. There were 41 male patients $(58.6 \%)$ and 29 female patients $(41.4 \%)$. The age of patients ranged from 40 to 82 years, average age was $63.5 \pm 2.06$ years. The average body mass index was equal to $28.03 \pm 0.33 \mathrm{~kg} / \mathrm{m}^{2}$.

Among the risk factors in patients with NSAID gastropathies, in $38(54.3 \%) \mathrm{H}$. pylori-infection was found, 22 patients $(31.4 \%)$ had smoking in their past medical history, 12 patients (17.1\%) consumed alcohol.
General clinical examination was performed to all patients, which included data of past medical history, laboratory tests, FGDS (fibrogastroduodenoscopy), stool-test to determine H. pylori.

The endogenous PGE2 serum content was investigated using ELISA reagent set PGE2 Immunoassay R\&D Systems on control calibration graph building and the received values comparing.

LTB4 plasma concentrations were determined using ELISA test kits LTB4 (Neogen corporation, USA) with the schedule construction of the calibration chart using the standard curve.

According to the study design, patients were divided into two groups, including 37 patients in the first group, who prophylactically received ASA in a dose of $75 \mathrm{mg}$ /day a long time. The second group included 33 patients with relevant disorders, which received aspirin $75 \mathrm{mg} /$ day and pantoprazole $40 \mathrm{mg} /$ day.

During FGDS, visual assessment of gastroduodenal region mucosa morphological pattern was performed. The attention was paid to the presence of hyperemia, edema, erosive and ulcerative lesions, duodenal bulb scar deformity, cardiac sphincter tone, presence of gastroesophageal reflux. Endoscopic changes in mucosa of the stomach and duodenum in patients with coronary heart disease, depending on the NSAIDs action duration were evaluated.

To verify the endoscopic degree evaluation of gastric mucosa destruction with NSAID gastropathies, Lanza score (2009) was used.

Statistical analysis included the comparison group which was conducted with the Mann-Whitney, Tau-Kendall coefficient. There were several research groups in this study, so the reliability of difference in patients treated with the different drugs, was determined by Newman-Keuls criterion, allowing unlike Student's $t$-test, Fisher conduct multiple analyzes.

\section{Results}

Clinical features of erosive and ulcerative lesions in 

NSAID Gastropathies Pantoprazole Treatment in Patients with Cardiovascular Pathology

the majority of patients with NSAID gastropaties were asymptomatic 46 (66\%). However, endoscopic examination revealed changes of the stomach and duodenum mucosa.

The results of study showed that the number of stomach erosive lesions decreased from $58.18 \%$ to $42.42 \%$, stomach ulcers from $14.55 \%$ to $6.06 \%$, duodenum erosive lesions decreased from $34.55 \%$ to $24.24 \%$, duodenum ulcers deceased from $9.09 \%$ to $3.03 \%$. In addition, hyperemia and bulbitis were recorded in the group of patients taking aspirin more than in the group treated with pantoprazole.

Therefore, pantoprazole significantly reduced the number of gastric ulcers $(p<0.05)$, gastric erosions $(p<0.01)$, duodenal erosions $(p<0.05)$, bulbite $(p<0.05)$.

For quantitative changes determination and mucosal lesions counting, Lanza score was used. It is based on an assessment of the erosions and ulcers number in one or more segments of the gastroduodenal zone.

The highest percentage of lesions was third grade Lanza scale - 12 (17\%) in patients taking aspirin, while patients taking pantoprazole-4 $(6 \%)$. Fifth grade of mucosal lesions in the aspirin group amounted 11 $(16 \%)$ when in those who received pantoprazole-3 (4\%). High percentage was first grade of lesions in patients taking aspirin and pantoprazole $11(16 \%)$ that was higher than in group treated aspirin only-4 (6\%). The similar result was at second grade on Lanza scale-8 $(11 \%)$ in aspirin group, $2(3 \%)$ in PPIs treated group. The identical percentage of the mucous membrane lesions fourth grade on Lanza scale was in both groups-8 (11\%) in aspirin, 7 (10\%) in pantoprazole and aspirin.

LTB4 and PGE2 levels learning was used for the determination opportunity of gastroduodenal mucosa aggressive and defensive factors, and for checking of its levels variation after aspirin and pantoprazole treatment. Also, it was interesting to watch of the Lanza scale regression after taking pantoprazole.

The LTB4 level in patients taking pantoprazole combined aspirin was lower than the patients treated aspirin only, namely 20 (12.75 46) (16.14 26.6) ng/ml in the first one which is significantly different from its range in the aspirin group $-50(30.5 \sim 60)(48 \sim 55)$ $(p<0.001)$.

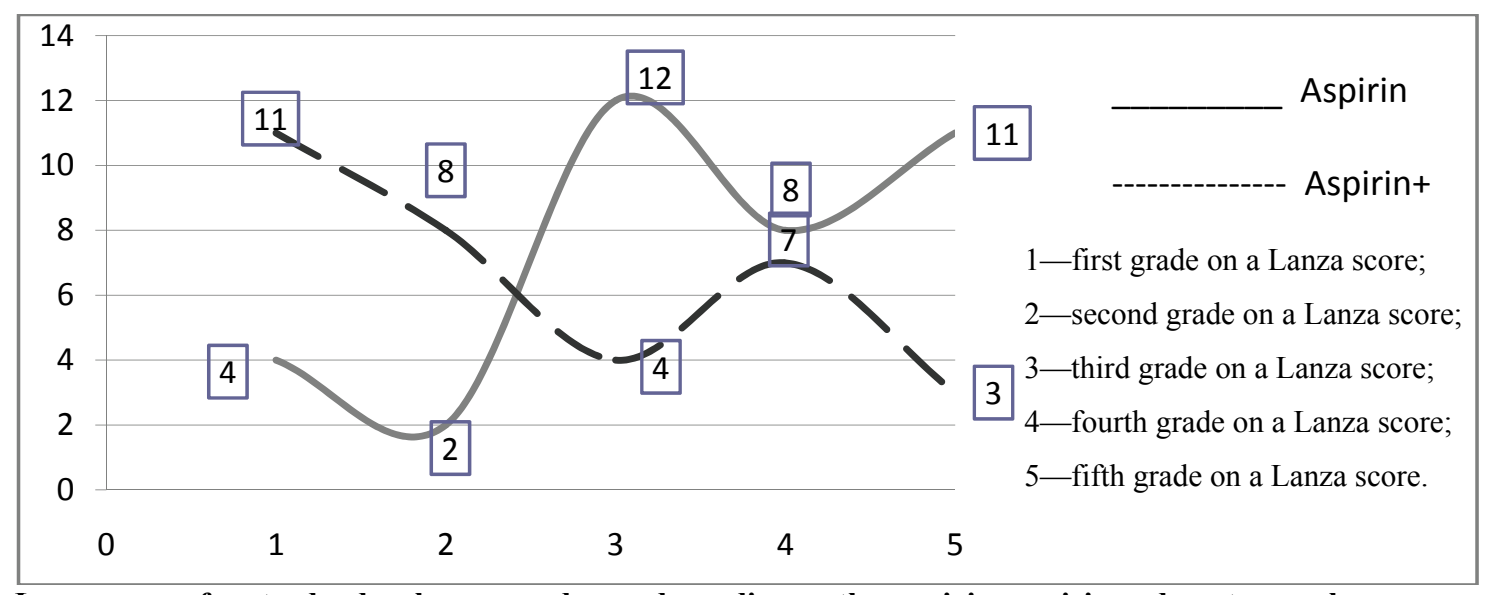

Fig. 1 Lanza score of gastroduodenal mucosae degree depending on the receiving aspirin and pantoprazole.

Table 1 LTB4 and PGE2 levels before and after pantoprazole treatment in patients with NSAID gastropaties.

\begin{tabular}{llll}
\hline Levels & Aspirin & Aspirin + Pantoprazole & $p$ \\
\hline \multirow{2}{*}{ LTB4 $(\mathrm{ng} / \mathrm{mL})$} & $50(30.5 \sim 60)$ & $20(12.75 \sim 46)$ & $<0.001$ \\
\hline \multirow{2}{*}{ PGE2 $(\mathrm{pg} / \mathrm{mL})$} & $48 \sim 55$ & $16.14 \sim 26.6$ & $>0.1$ \\
\hline \multirow{2}{*}{ Lanza score } & $1,400(850 \sim 3,400)$ & $\begin{array}{l}1,260(440 \sim 1,870 \\
1,110 \sim 1,600\end{array}$ & 0.015 \\
\hline
\end{tabular}


Table 2 LTB4 and PGE2 correlation on Lanza score after pantoprazole treatment.

\begin{tabular}{lll}
\hline Items & Tau-Kendall & $p$-level \\
\hline Lanza score\&LTB4 & 0.32 & $<0.0001$ \\
Lanza score\&PGE2 & -0.04 & $>0.05$ \\
\hline
\end{tabular}

When comparing the performance PGE2 in patients with NSAID gastropathy and a group of patients who were administered the treatment pantoprazole $40 \mathrm{mg}$ for one month, no significant difference was noted in terms of PGE2 $(p>0.05)$. PGE2 content ranges from $440 \mathrm{pg} / \mathrm{mL}$ to $1,870 \mathrm{pg} / \mathrm{mL}$, on average amounted to $1,260 \mathrm{pg} / \mathrm{mL}$ in the aspirin and PPI treated group and from 850 to $3,400 \mathrm{pg} / \mathrm{mL}$, on average $1,400 \mathrm{pg} / \mathrm{mL}$, in the group who used to took aspirin.

Consequently, the pantoprazole course assignment in patients with NSAIDs gastropathies occurred a significant reduction of LTB4 $(p<0.01)$, without no significant changes in PgE2 content.

Also, it was investigated that the degree of lesions on the Lanza score was lower in patients taking pantoprazole $(p<0.01)$.

To confirm reliably the importance of the relationship and LTB4 and PGE2 definition, its correlation was analyzed with the Lanza score by Tau-Kendall coefficient

The positive correlation between LTB4 level and Lanza score was marked. It indicates that the increasing of gastroduodenal mucosal lesion area severity could significantly raise the LTB4 level. It could be an important marker for the NSAIDs gastropathy diagnosis.

On the other hand, correlation between PGE2 and Lanza score was not found. It means, the pantoprazole action mechanism is associated with LTB4 inhibition, which induces the healing of erosive ulcerous defects.

\section{Discussion}

The cause of gastroduodenal erosive and ulcerative lesions, and bleeding and perforations, associated with it, is NSAIDs receiving, including aspirin $[3,4,11]$.

The main action mechanism of NSAIDs damaging on the stomach and duodenum mucous membrane is its cyclooxygenase (COX) enzyme blockade, which has two isomers: block COX-1 and COX-2 induced $[4,5,7]$. Inhibition of COX-1 activity, while taking NSAIDs, causes depression of prostaglandin synthesis system, which serves as the main cause of such side effects as gastroduodenal erosive and ulcerative lesions. It deplets the stocks in the tissues and causes iatrogenic prostaglandins failure [3,5].

Interacting with the binding site of COX-1, NSAIDs interrupt the arachidonic acid cyclooxygenase pathway, which prevents the synthesis of prostaglandin, thromboxane and prostacyclin [9]. NSAIDs, that are weak organic acids, easily penetrate the phospholipid membrane into the cells cytoplasm, causing local damage to the mucosa-erosions and ulcers [7].

In the destructive processes mechanism of the stomach and duodenum mucous membrane, the important role is by the leukotriene B4 and prostaglandin E2 correlation [9].

The violation of its, towards the LTB4 predominance, causes local inflammation and gastric mucosa toxic injuring, induces the adhesion and active neutrophil chemotaxis on epithelial cells. It damages the microcirculatory source in the stomach lamina propria mucosa $[7,9]$.

The drugs using for parenteral and rectal administration, reduces the erosive and ulcerative lesions risk only in the first 7 14 days of treatment. Then, NSAIDs cause the systematic suppression of prostaglandin synthesis, regardless of the drug and the ways of getting in $[1,4]$.

PPIs have been reported to inhibit radical production and inflammation by suppressing the neutrophil activity [8]. The study results suggest that pantoprazole has a mucosa-protecting effect on the healing process of erosions and ulcers by decreasing the LTB4 

NSAID Gastropathies Pantoprazole Treatment in Patients with Cardiovascular Pathology

production, but without significant increasing PGE2.

\section{Conclusions}

The survey results complement the current issues about the mechanism of stomach and duodenal erosive and ulcerative lesions development on influence of NSAIDs. Diagnostic significance of LTB4 and PGE2 determination in patients with NSAIDs gastropaties combined with coronary heart disease was proven. An important factor which contributes the gastric and duodenal mucosa injuringin the ASA appointment is the LTB4 and PGE2 violation. It was investigated that the erosive and ulcerative lesionshealing depends on LTB4 level decreasing after pantoprazole treatment. However, its effect was not proved on the PGE2 ratio, which requires gastrocytoprotectors adding to NSAIDs management.

\section{References}

[1] Lanas, A. 2011. "Low Doses of Acetylsalicylic Acid Increase Risk of Gastrointestinal Bleeding in a Meta-analysis" Clinical Gastroenterology and Hepatology 9: 762-8.

[2] Iwamoto, J., Saito, Y., Honda, A., and Matsuzaki, Y. 2013. "Clinical Features of Gastroduodenal Injury associated with Long-Term Low-Dose Aspirin Therapy." World Journal of Gastroenterology 19 (11): 1673-82.

[3] Wallace, J. L. 2001. "Pathogenesis of NSAID-Induced Gastroduodenal Mucosal Injury." Best Practice\&Research Clinical Gastroenterology 15:
691-703.

[4] Melcarne, L., García-Iglesias, P. and Calvet, X. 2016. "Management of NSAID-associated Peptic Ulcer Disease." Expert Review of Gastroenterology and Hepatology 1: 11.

[5] Kim, H. M., Cho, J. H., Cho, J. Y., Chun, S. W., and Kim, Y. J. 2013. "NSAID Is Inversely associated with Asymptomatic Gastric Ulcer: Local Health Examination Data from the Korean National Health Insurance Corporation Scandinavian.” Journal of Gastroenterology 48 (12): 1371-6.

[6] Laine, L. 2001. "Approaches to Nonsteroidal Anti-inflammatory Drug Use in the High-Risk Patient." Gastroenterology 120: 594-606.

[7] Kopáčová, M., Bureš, J. and Tachecí, I. 2013. “Video Gastrointestinal Lesions Detected by Capsule Endoscopy and Double-Balloon." Enteroscopy Journal and Encyclopedia of GI Endoscopy 1: 180-2.

[8] Mo, C., Sun, G., Lu, M., Zhang, L., Wang, Y. Z., Sun, X. and Yang, Y. S. 2015. "Proton Pump Inhibitors in Prevention of Low-Dose Aspirin-Associated Upper Gastrointestinal Injuries." World Journal of Gastroenterology 21 (17): 5382-92.

[9] Okazaki, M., Shimizu, I., and Ishikawa, M. 2007. "Gastric Mucosal Levels of Prostaglandins and Leukotrienes in Patients with Gastric Ulcer after Treatment with Rabeprazole in Comparison to Treatment with Ranitidine." Journal of Medical Investigation 54: 83-90.

[10] Wallace, J. L. 2008. "Prostaglandins, NSAIDs, and Gastric Mucosal Protection: Why Does not the Stomach Digest Itself?." Physiological Reviews 88 (4): 1547-65.

[11] Zhu, L. L., Xu, L. C., Chen, Y., Zhou, Q., and Zeng, S. 2012. "Poor Awareness of Preventing Aspirin-Induced Gastrointestinal Injury with Combined Protective Medications." World Journal of Gastroenterology 18 (24): 3167-72. 